\title{
Synthesis of Organofunctionalized Silica Gel from Tributylstannylated Silicic Acid and Acyl Chloride
}

\author{
Takahisa IIDA, Toshifumi Kageyama, Toshio Sugizaki, ${ }^{*}$ and Osamu MoriYa**† \\ Department of Industrial Chemistry, Faculty of Engineering, Kanto Gakuin University, \\ Mutsuura, Yokohama 236, Japan \\ *Lintec Co., Ltd., Nishikicho, Warabi, Saitama 335, Japan \\ ** Department of Chemistry, National Defense Academy, \\ Hashirimizu, Yokosuka 239, Japan
}

(Received March 27, 1997)

\begin{abstract}
KEY WORDS Organofunctionalized Silica Gel / Acyl Chloride / Tributylstannylated Silicic Acid / Silanol / Water Glass /
\end{abstract}

Silica gels modified with organofunctional groups are used widely as industrial materials. The broadened usage such as hybrid materials, immobilization of enzymes, and convenient catalysts requires various synthetic methods of the functionalized gel. ${ }^{1,2}$ The straightforward reaction of silica gel and organic compounds is generally utilized for the modification as a practical procedure. In addition, the use of oligomeric and/or monomeric silanols obtained from sodium metasilicate or water glass (WG) through neutralization has been investigated as a convenient route to organofunctionalized silica gel. ${ }^{3-8}$ However, such silanols are readily transformed into silica gel by selfcondensation. This sometimes causes difficulties to handle in organofunctionalization. Therefore, recently, the protected monomeric silanols, tetraalkoxysilanes, which are free from the above problems, are often used for incorporation of organic groups into silica gel. ${ }^{9} \mathrm{We}$ have investigated a new versatile precursor of functionalized silica gel and postulate that tributylstannyl ester of silicic acid (PTBS), the protected polymeric silanol, is a possible candidate. ${ }^{10}$

The use of PTBS having Sn-O bond, which is soluble in common organic solvents such as hexane, benzene, dichloromethane, and chloroform, should make possible efficient reactions with organic compounds. It is well known in organotin chemistry that stannyl ethers having $\mathrm{Sn}-\mathrm{O}$ bond react with acyl halides easily to afford esters and organotin halide. ${ }^{11,12}$ In a similar manner, the reaction of acyl chlorides and PTBS having $\mathrm{Si}-\mathrm{O}-\mathrm{Sn}$ bonds is thought to form $\mathrm{Si}-\mathrm{OC}=\mathrm{O}$ bonds with the elimination of tributyltin chloride as shown in Scheme 1. Such reaction seems to represent the incorporation of an organofunctional group into silica gel by the use of PTBS. On the other hand, $\mathrm{Si}-\mathrm{O}$ bond is known to be more stable against halogenation than $\mathrm{Sn}-\mathrm{O}$ bond. ${ }^{13}$ However, the possibility of the formation of $\mathrm{Si}-\mathrm{Cl}$ bond, as shown in the reaction of trimethylsilylated silica gel with a well known halogenating agent such as thionyl chloride, is not thoroughly excluded. ${ }^{6}$ In this type of reaction, the formation of chlorinated silica gel and tributyltin ester is presumed. Accordingly, with interest in chemoselectivities of the reaction at $\mathrm{Si}-\mathrm{O}-\mathrm{Sn}$ bond, the reaction of PTBS and acyl chlorides is examined as a new approach toward the synthesis of organofunctionalized silica gel in this work.

\section{EXPERIMENTAL}

\section{General Procedure}

PTBS was prepared from bis(tributyltin) oxide and water glass according to the reported procedure. The molecular weight $\left(M_{n}\right)$ of PTBS was estimated by GPC (polystyrene standard) to be in the region from 3000 to $15000 .^{10}$ The ratio of $\mathrm{Si} / \mathrm{Sn}$ in PTBS determined by gravimetric analysis was $c a .2 .7$.

The reactions of PTBS with acyl chlorides 1 or diacyl chloride 3 were carried out at room temperature for $6 \mathrm{~h}$ in benzene, dimethylformamide (DMF), or a mixture of acetonitrile and hexane (50/50, vol\%). Double the molar quantity of 1 to tributyltin group in PTBS was used except for one example (Run 2), in which the reaction was conducted at $80^{\circ}$ with an equimolar amount of $\mathbf{1}$. In reactions using diacyl chlorides 3 , an equimolar
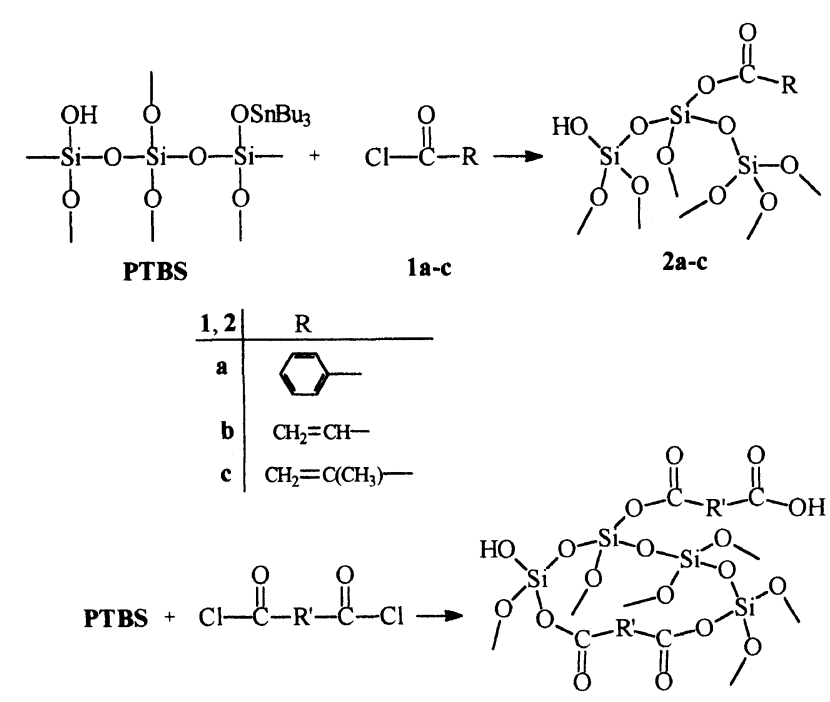

3a-b

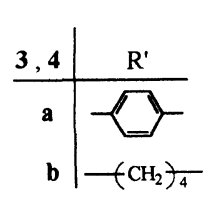

4a-b

\footnotetext{
$\dagger^{\dagger}$ To whom all correspondence should be addressed.
} 
Table I. Preparation of organofunctionalized silica gels 2 and $\mathbf{4}$ from PTBS and acyl chloride 1

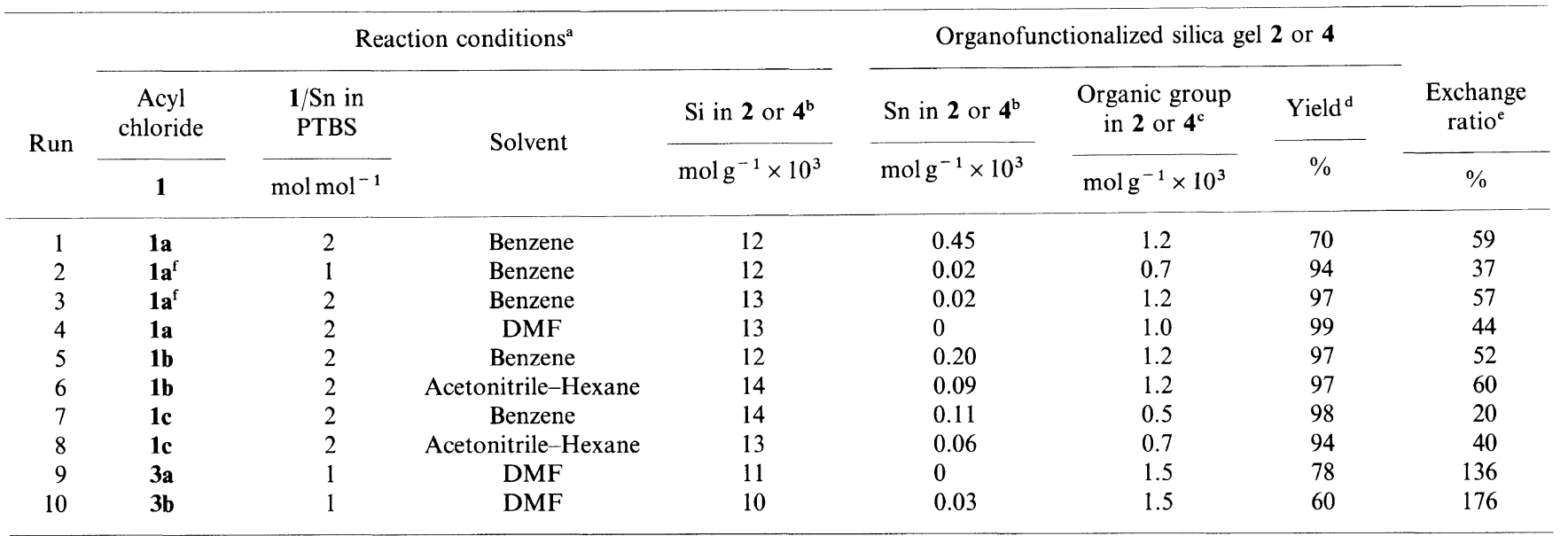

${ }^{\mathrm{a}}$ The reactions were carried out at room temperature except for Runs 2 and $3 .{ }^{\mathrm{b}}$ Estimated from gravimetric analysis. ${ }^{\mathrm{c}}$ Estimated from gravimetric and elemental analysis. ${ }^{\mathrm{d}}$ Based on Si in PTBS and $\mathbf{2}$ or $\mathbf{4}$. $^{\mathrm{e}}$ Calculated according to the following equation: $\{$ Incorporated organic group $(\mathrm{mol}) /$ Eliminated $\mathrm{Sn}(\mathrm{mol})\} \times 100 .{ }^{\mathrm{f}}$ The reaction was carried out under reflux in benzene.

amount of 3 to $\mathrm{Sn}$ unit was employed, i.e., the ratio of acyl chloride group to tin unit was adjusted to be 2 . After the reaction, the precipitated gel was filtered and washed with dimethylformamide, water, benzene, and diethyl ether and then dried 1 day at $70^{\circ} \mathrm{C}$ under reduced pressure such as $10 \mathrm{mmHg}$. These results of the reactions are listed in Table I. Yields were based on the content of Si, determined by gravimetric analysis, in starting PTBS and the functionalized silica gel 2 . The contents of organic groups in $\mathbf{2}$ were estimated by the results of elemental and gravimetric analysis, in which the number of carbons was assigned to the functional group and remained tributyl group of tin. The ratio of incorporated organic group and eliminated tributyltin group was calculated as the exchange ratio.

\section{Gravimetric Analysis}

A sample was oxidized by nitric acid at $60^{\circ} \mathrm{C}$ under atmospheric pressure in a platinum crucible until an liquid part was vaporized out. After drying at $600^{\circ} \mathrm{C}$ for $2 \mathrm{~h}$, the weight of the resulting precipitates, which were regarded as the mixture of $\mathrm{SiO}_{2}$ and $\mathrm{SnO}_{2}$, was measured. The precipitates were treated with a mixture of conc. hydrofluoric acid and conc. nitric acid (50/50, vol\%) similarly to the oxidizing step. The content of $\mathrm{SiO}_{2}$ was calculated from the weight loss of these two steps and the weight of the residual precipitate was regarded as that of $\mathrm{SnO}_{2}$.

\section{RESULTS AND DISCUSSION}

From the reaction of PTBS and double the molar amount of benzoyl chloride 1a to tributyltin group in benzene at room temperature, the silica gel $\mathbf{2 a}$ was obtained in $70 \%$ yield, but the tributyltin group still remained in the product $\mathbf{2 a}$ (Run 1). The reactions proceeded more effectively at $80^{\circ} \mathrm{C}$ and almost all tributyltin group in PTBS was eliminated even in the example using an equivalent of 1a to $\mathrm{Sn}$ group (Run 2). 2a was also obtained quantitatively and the tributyltin group was thoroughly eliminated in the reaction using DMF as solvent, in which PTBS was not dissolved completely (Run 4). This suggests that a polar solvent

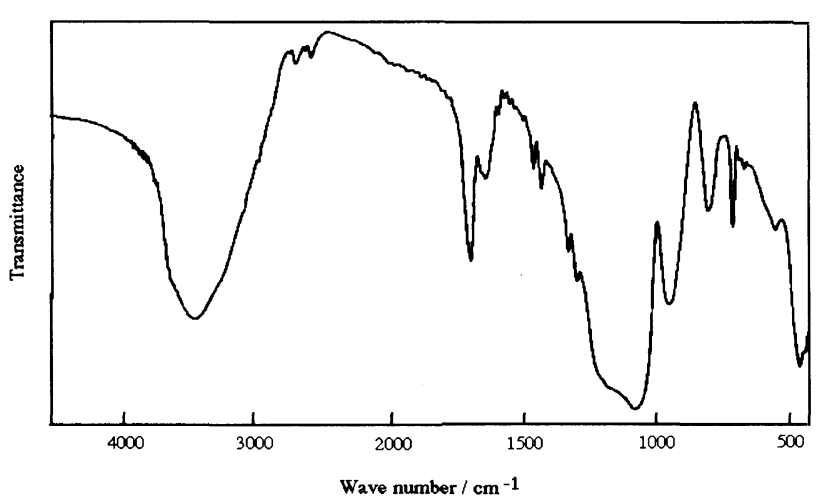

Figure 1. IR spectrum of organofunctionalized silica gel $\mathbf{2 a}$.

affected the reaction of tributyltin group and acyl chloride even in a heterogeneous reaction system. This speculation was supported by the results obtained from the reactions using the mixed solvents of acetonitrile as a polar solvent and hexane, in which tin group was also eliminated efficiently before gelation (Runs 6 and 8). In these cases, PTBS was not soluble in acetonitrile and acyl chloride was not in hexane.

The contents of organic group in the gel 2 were varied from $0.70 \mathrm{mmol} \mathrm{g}^{-1}$ to $1.2 \mathrm{mmol} \mathrm{g}^{-1}$ in all cases using acyl chloride 1 . The exchange ratios were found in the region from $40 \%$ to $60 \%$ except for one example (Run 7). These results suggest that almost half the Si unit bonded with the stannyloxy group participated in the substitution reaction and the rest of the unit took part in the gelation. The formation of $\mathrm{Si}-\mathrm{OC}=\mathrm{O}$ bond was confirmed by IR spectra of 2 , in which absorption assigned to $\mathrm{C}=\mathrm{O}$ bond was observed around $1700 \mathrm{~cm}^{-1}$. In the IR spectra of 2 , the absorption around $2900 \mathrm{~cm}^{-1}$ due to tributyl group of tin was not found or became small. Absorption at $1100 \mathrm{~cm}^{-1}, 790 \mathrm{~cm}^{-1}$, and $475 \mathrm{~cm}^{-1}$ assigned to $\mathrm{Si}-\mathrm{O}-\mathrm{Si}$ bond was observed in both IR spectra of PTBS and 2. IR spectrum of $\mathbf{2 a}$ is shown in Figure 1.

The reactions of PTBS and diacyl chlorides 3 , expected to affect advantageously cross-linkage of Si unit, were conducted at room temperature for $6 \mathrm{~h}$ in DMF solution. From the reactions of PTBS with terephthaloyl dichloride $\mathbf{3 a}$ and adipoyl dichloride $\mathbf{3 b}$, the gels $\mathbf{4}$ having 


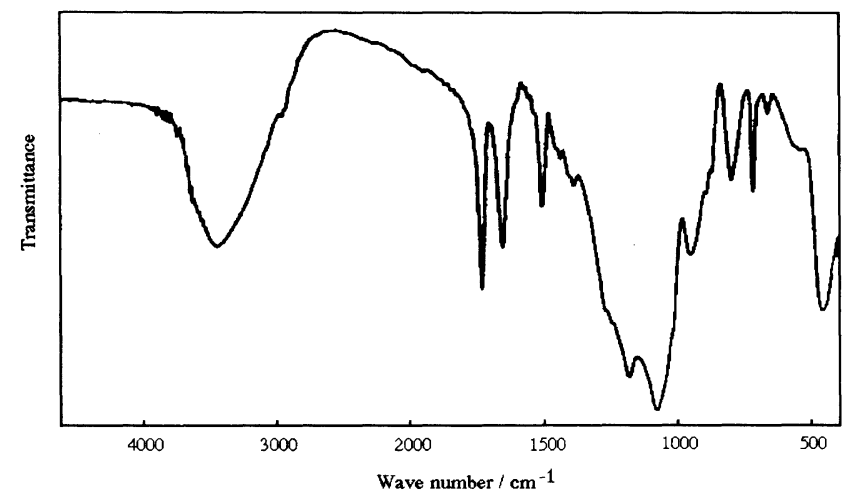

Figure 2. IR spectrum of organofunctionalized silica gel $\mathbf{4 a}$.

terephthalic and adipic group were obtained in $60 \%$ and $78 \%$ yield (Runs 9 and 10). Exchange ratios were calculated to be $136 \%$ and $176 \%$, respectively, in the reactions using an equivalent of $\mathbf{3 a}$ and $\mathbf{3 b}$ to tributyltin unit in PTBS. The calculated ratio was based on the assumption that two tributyltin groups react with one diacyl chloride 3. Therefore, such observation of values of over $100 \%$ suggests the possibility that one of acyl chloride groups in 3 reacted with tributyltin group and the other remained without substitution. The two characteristic absroptions due to carbonyl groups observed at $1680 \mathrm{~cm}^{-1}$ and $1730 \mathrm{~cm}^{-1}$ in the IR spectrum of $4 a$, as shown in Figure 2, seems to support such speculation.

Although more detailed experiments are required to clarify the exact product selectivity of the reaction to silyl ester, it may be said at present that the formation of $\mathrm{Si}-\mathrm{OC}=\mathrm{O}$ bond proceeds by the use of almost half of $\mathrm{Si}-\mathrm{O}-\mathrm{Sn}$ bonds in PTBS. Tributyltin chloride was isolated by distillation in $70 \%$ yield from the filtrate obtained in the reaction using benzoyl chloride (Run 2).
However, it was difficult by our method to clarify the formation of chlorinated silica gel, which should be formed in the other reaction, since, dechlorination from silica gel is thought to occur readily by washing the resulting gel with water and alcohol. ${ }^{6}$

The incorporation of organofunctional group into silica gel by acyl chlorides with a base has been reported. ${ }^{14}$ In comparison with such reaction, the use of PTBS is thought to be another efficient approach, in which the reaction with acyl chloride readily proceeds by just mixing these reagents at room temperature.

\section{REFERENCES}

1. R. Kroker, M. Schneider, and K. Hamann, Prog. Org. Coatings, 1 (1972).

2. K. Haraguchi and Y. Usami, Chem. Lett., 51 (1997).

3. Y. Abe, in "Muki Kobunshi," M. Kajiwara and K. Murakami, ed., Sangyo Tosho, Tokyo, 1992, Chapter 2.

4. J. Götz and C. R. Masson, J. Chem. Soc., (A), 2683 (1970).

5. H. Inoue and S. Kohama, J. Polym. Sci., Polym. Lett. Ed., 18, 795 (1981).

6. R. Currel and J. R. Parsonage, Macromol. Sci.-Chem., A16(1), 141 (1981).

7. Y. Abe and T. Misono, J. Polym. Sci., Polym. Lett. Ed., 22, 565 (1984).

8. Y. Abe, A. Kaijou, N. Shintani, Y. Nagao, and T. Misono, $J$. Polym. Sci., Part A, Polym. Chem., 25, 1671 (1987).

9. S. Sakka, in "Treatise on Matearials Science and Technology 22, Glass III," M. Tomozawa and R. Doremus, Ed., Academic Press, New York, N.Y., 1982, p 129.

10. T. Iida, T. Sekiya, T. Kageyama, T. Sugizaki, and O. Moriya, Chem. Lett., 1947 (1996)

11. R. Okawara, D. G. White, K. Fujitani, and H. Sato, J. Am. Chem. Soc., 83, 1342 (1961).

12. S. Sakai, K. Ito, and Y. Ishii, Yuki Gosei Kagaku Kyokai Sci., 28, 1109 (1970).

13. K. Itoh, A. Nozawa, and Y. Ishii, Tetrahedron Lett., 1421(1969).

14. J. Wartmann and H. Deuel, Helv. Chim. Acta, 42, 1160 (1959). 